\title{
Aktivitäten von Akteuren in Schweizer Bildungslandschaften - Welchen potenziellen Beitrag zu mehr Chancengerechtigkeit könnten sie leisten?
}

\author{
Anja Koszuta $(\mathbb{D} \cdot$ Ricarda Werner $\cdot$ Stephan G. Huber
}

Eingegangen: 17. September 2020 / Überarbeitet: 15. April 2021 / Angenommen: 22. April 2021 / Online publiziert: 14. Juni 2021

(C) Der/die Autor(en) 2021

Zusammenfassung In diesem Artikel werden zunächst theoretische Überlegungen zu möglichen gerechtigkeitsbezogenen Unterstützungsleistungen durch Bildungslandschaften für das Schulsystem angestellt. Anschließend werden diese Überlegungen mit den Aktivitäten innerhalb von Bildungslandschaften anhand einer Dokumentenanalyse in Bezug gesetzt. Ziel des Papers ist zu untersuchen, welche Gerechtigkeitsdimensionen durch die Maßnahmen, die durch die Bildungslandschaften entstanden sind, berücksichtigt werden. Als Datengrundlage gelten Dokumente, die im Rahmen der Berichterstattung an die geldgebende Stiftung (z. B. Zwischenreportings über den aktuellen Stand des Projekts, Schlussberichte) sowie Dokumentationen, Flyern u. ä. zu Massnahmen, die in vereinzelten Bildungslandschaften entstanden sind. Die Befunde verdeutlichen, dass die Aktivitäten von Bildungslandschaften vor allem Aspekte der Integrationskraft berühren. Hierzu zählen auf Ebene der Bevölkerung die Schaffung von Austausch- und Begegnungsgelegenheiten und informierenden Angebote sowie die Sichtbarmachung vorhandener Angebote und auf Ebene der Akteure netzwerksbildungsfördernde Maßnahmen. Weiterhin wird deutlich, dass sich die Mehrheit der Aktivitäten an die Bevölkerung und weniger an die Fachkräfte des Bildungssystems richtet.

Schlüsselwörter Bildungsnetzwerke $\cdot$ Bildungsangebote $\cdot$ Auswirkungen von Bildungslandschaften · Soziale Ungleichheit · Dokumentenanalyse

Anja Koszuta $(\square) \cdot$ Ricarda Werner $\cdot$ Stephan G. Huber

Pädagogische Hochschule Zug, University of Teacher Education Zug,

Zugerbergstrasse 3, 6300 Zürich, Schweiz

E-Mail: anja.koszuta@hotmail.com 


\title{
Activities in educational networks - In what ways do they consider dimensions of social equity? Qualitative findings of a document analysis
}

\begin{abstract}
In a first step this paper reflects theoretically on the potential equityrelated support of educational networks. In a second step, these reflections are put into effect with the activities of educational networks within the framework of a document analysis. The aim of this paper is to explore, in what ways the activities in educational networks consider the dimensions of social equity. Our analysis draws on data gathered from networks in German- and French-speaking Switzerland. Information about activities of these learning networks were collected from project reportings and (if available) from websites, brochures etc. Findings show that both the network activities as well as the educational activities in educational networks consider dimensions of equity of educational opportunities. The majority of activities of educational networks was assigned to the dimension integration. This dimension includes the installation of meeting places, providing the parents and youth with information that are needed for participating in society as well as making educational offers more visible. The analysis also shows that most of the educational activities of educational networks are aimed at the population and less educational offers are aimed at staff in the educational system.
\end{abstract}

Keywords Educational arrangements - Educational offers - Impact of educational networks $\cdot$ Social inequality $\cdot$ Document analysis

\section{Einleitung}

Seit dem Jahr 2000 haben die PISA-Ergebnisse wiederholt belegt, dass die Bildungs- und folglich auch die Berufschancen in der Schweiz stark von der sozialen Herkunft abhängen (Konsortium PISA.ch 2018). Gleichzeitig macht die Transformation moderner Gesellschaften in Wissensgesellschaften eine umfassende Bildung aller Individuen erforderlich, damit diese den steigenden Anforderungen der Arbeitswelt gerecht werden können (Bell 1999; Hradil 2006). Die Gesamtheit der Bildungsprozesse eines Individuums ergibt sich hierbei nicht nur aus der schulischen Bildung, sondern aus dem Zusammenspiel von formaler, non-formaler und informeller Bildung (Fend et al. 2004). Zudem erfolgt die Betreuung und Bildung von Kindern und Jugendlichen auch in der Schweiz immer häufiger außerhalb der Familie (SKBF 2018). Im internationalen Kontext wurde diesen Entwicklungen mit verschiedensten Reformbemühungen begegnet, die sowohl zu einer höheren Bildungsbeteiligung als auch zu einer größeren Chancengerechtigkeit beitragen sollen. Hierzu zählt auch der Aufbau sogenannter Bildungslandschaften, also eine zunehmenden Vernetzung von schulischen und außerschulischen Bildungsakteuren (Huber 2014). Das Ziel von Bildungslandschaften besteht darin, Bildungseinrichtungen zu vernetzen und durch verstärkte Kooperationen Kindern und Jugendlichen sowohl bessere Bildungsbedingungen als auch vielfältigere Bildungsmöglichkeiten zu bieten. Dieses breit gefächerte und konzeptionell verbundene Bildungs-, Betreuungsund Freizeitangebot soll zum einen eine verlässliche Betreuung von Kindern und 
Jugendlichen gewährleisten sowie einen Beitrag zur Verringerung bestehender Bildungsungleichheiten leisten (BJK 2002; BMFSFJ 2005).

Die Erfahrungen aus anderen Ländern (u.a. Deutschland, Niederlande) lassen den vorsichtigen Schluss zu, dass die Zusammenarbeit von schulischen und außerschulischen Bildungsakteuren nicht nur positive Auswirkungen auf die Akteure und deren Arbeit (Bauer et al. 2017), sondern auch auf die Qualität der Bildungsangebote und damit verbunden auch auf den Lernzuwachs der Kinder und Jugendlichen hat (Rolff 2014). Die Befundlage weist jedoch international einige Lücken auf und ist für die Schweiz überhaupt noch nicht vorhanden. Zudem werden gerechtigkeitstheoretische Ansätze kaum explizit in die Untersuchungen mit einbezogen. Daran anschließend untersucht das vorliegende Paper, welche Gerechtigkeitsdimensionen mit den einzelnen Maßnahmen, die innerhalb einer Bildungslandschaft angeboten wurden, berücksichtigt werden.

\section{Bildungslandschaften im Bildungsbereich}

Bei dem Begriff Bildungslandschaft handelt es sich um ein Konzept aus der Bildungspolitik, dem ein ganzheitliches Bildungsverständnis zugrunde liegt, und der eine strategische Allianz verschiedener Behörden sowie schulischer und außerschulischer Einrichtungen zur Gestaltung von Bildungsbiografien, die in der Regel die Zeitspanne vom Kindergartenalter bis zur Beendigung von Studium oder Berufsausbildung junger Erwachsener umfassen, bezeichnet (Huber 2014; Huber et al. 2014). Je nach Kooperationsanlass arbeiten die relevanten Einrichtungen in einer überschaubaren, räumlichen Nähe zusammen, z. B. innerhalb einer Gemeinde. Seit den 2000er Jahren wurden sowohl national als auch international zahlreiche Reforminitiativen, die den Aufbau von Bildungslandschaften unterstützt haben, umgesetzt. Ähnlich wie beim deutschsprachigen Diskurs um Ganztagsschulen geht auch die Einführung von Bildungslandschaften mit der Hoffnung auf einer Verbesserung der Bildungsgerechtigkeit einher (BJK 2002; BMFSFJ 2005). So werden denn auch von den meisten Bildungslandschaften eine verbesserte Bildungsteilhabe und Chancengerechtigkeit als Zielsetzung formuliert (Jungermann et al. 2015). Auch wenn Bildungslandschaften in den deutschsprachigen Ländern sukzessive - sowohl in der Praxis als auch in der Forschung - einen Zuwachs erfahren, ist das Konzept der Bildungslandschaft noch relativ jung und ihre Erforschung steht noch am Anfang. Mit der Veröffentlichung von Ergebnissen der wissenschaftlichen Begleitforschung einzelner Reforminitiativen (z. B. Schulen im Team, Selbstständige Schule, Lernende Regionen - Netzwerke gestalten) erschienen in den vergangenen Jahren jedoch wichtige Forschungsberichte, die Hinweise zu Aspekten der Kooperation, Gelingensbedingungen und Wirkungen bei der Entwicklung von Bildungslandschaften geben. Im deutschsprachigen Forschungsdiskurs zu Bildungslandschaften dominieren zum einen v. a. governanceanalytische Untersuchungen, die Unterstützungsleistungen von intermediären Steuerungsformen, z. B. regionalen Bildungsbüros, untersuchen (Berkemeyer et al. 2009; Järvinen et al. 2015; Manitius 2013; Manitius et al. 2015). Zum anderen werden Kooperationsaspekte betrachtet. Zusammenfassend kann formuliert werden, dass Bildungslandschaften nicht nur quantitativ gesehen zu mehr 
Zusammenarbeit zwischen schulischen und außerschulischen Akteuren führen, sondern diese auch qualitativ verbessern können. Durch die verstärkte Zusammenarbeit kann das gegenseitige Verständnis füreinander verbessert und ein gemeinsames Bildungsverständnis entwickelt werden (Schalkhausser und Thomas 2011).

Weiterhin konnten finanzielle Ressourcen, gegenseitiges Vertrauen sowie eine Aushandlungs- und Partizipationskultur als Gelingensbedingungen identifiziert werden (Huber 2014; Huber et al. 2019; Maag Merki et al. 2009; Stolz 2009; Tippelt et al. 2011). Schließlich stehen auch die Wirkungen von Bildungslandschaften im Fokus einiger Untersuchungen. Auf Schulebene weisen bisherige Forschungsarbeiten zum Beispiel auf eine Zunahme von interdisziplinären Kooperationen hin (Schubert et al. 2011). Gemäß Rolff (2014) führt eine verstärkte Vernetzung der Schulen mit verschiedenen Kooperationspartnern zudem nachweislich zu einer Verbesserung der Angebotsqualität. Auf Ebene der Bildungsakteure konnte ein besseres Verständnis der Akteure unterschiedlicher Bildungsinstitutionen füreinander sowie eine bessere Nutzung vorhandener Ressourcen bzw. eine Gewinnung von weiteren Ressourcen beobachtet werden (Bauer et al. 2017; Rolff 2014).

Im bisherigen Forschungsdiskurs werden Bildungslandschaften und weitere Regionalisierungsmaßnahmen jedoch selten in Bezug zu ihrer häufigsten Zielsetzung - nämlich die Verbesserung von Chancengerechtigkeit - gesetzt. Eine Ausnahme findet sich jedoch bei Duveneck (2016), die in ihren Analysen die Reproduktion von Wettbewerbslogiken aufzeigt und daraus eher eine Erhaltung des Status Quo bzw. eine Verstärkung der Ungleichheitsstrukturen ableitet. Die explizite Einbindung von gerechtigkeitstheoretischen Ansätzen nimmt in bisherigen Arbeiten jedoch eher eine untergeordnete Rolle ein. Neben den Ausführungen von Berse (2009) stellt die Arbeit von Manitius et al. (2015), die zunächst theoretische Überlegungen zu möglichen gerechtigkeitsbezogenen Unterstützungsleistungen durch Regionalisierungsmaßnahmen für das Schulsystem anstellt, eine Ausnahme dar. Anschließend werden diese Überlegungen mit den Leistungen von Regionalen Bildungsbüros in NordrheinWestfalen in Bezug gesetzt. Sie kommen zu dem Ergebnis, dass die Aktivitäten dieser Bildungsbüros insbesondere auf die Unterstützung der Durchlässigkeit von Schulsystemen abzielen (Ebd.). Die vorliegende Untersuchung knüpft an die Überlegungen von Manitius et al. (2015) an, die in ihren gerechtigkeitstheoretischen Ausführungen auf den Chancenspiegel - einem Instrument für Schulmonitoring zurückgreifen.

\section{Theoretischer Zugang zu Chancengerechtigkeit des Schulsystems}

In einer modernen Gesellschaft erfolgt die Legitimation sozialer Unterschiede nach dem meritokratischem Prinzip (Becker 2009). In den vergangenen zwei Jahrzehnten haben die PISA-Ergebnisse jedoch wiederholt belegt, dass die Bildungschancen in der Schweiz stark von der sozialen Herkunft abhängig sind (Konsortium PISA.ch. 2018). Vor diesem Hintergrund ist es bedeutsam, die Entwicklung des Schulsystems gerechtigkeitstheoretisch $\mathrm{zu}$ beobachten.

Basierend auf den drei Konzeptionen von Bildungsgerechtigkeit von Rawls, Sen und Honneth in Verbindung mit schultheoretischen Ansätzen haben Berkemeyer 
et al. (2012) für die Konzeptionalisierung und Auswertung der Chancengerechtigkeit des Schulsystems den Chancenspiegel entworfen. Berkemeyer et al. (2012) fassen Chancengerechtigkeit als ,die faire Chance zur freien Teilhabe an der Gesellschaft, die auch gewährleistet wird durch eine gerechte Institution Schule, in der Schülerinnen und Schüler aufgrund ihrer sozialen und natürlichen Merkmale keine zusätzlichen Nachteile erfahren, durch eine Förderung der Befähigung aller und durch eine wechselseitige Anerkennung der an Schule beteiligten Personen"1 (Berkemeyer et al. 2012 2017). Diese Auffassung von Chancengerechtigkeit beinhaltet sowohl die Überwindung von Nachteilen als auch die Entwicklung von Potenzialen. Insgesamt wird die Gerechtigkeit von Schulsystemen auf der Basis von vier zentralen Gerechtigkeitsdimensionen untersucht: Integrationskraft, Durchlässigkeit, Kompetenzförderung, Zertifikatsvergabe. Die Entwicklung dieser Gerechtigkeitsdimensionen basieren zum einen auf den gerechtigkeitstheoretischen Dimensionen des Verteilungs- (Rawls 2003), Befähigungs- (Sen 2005) und Anerkennungsansatzes (Honneth 2011) und zum anderen auf schultheoretischen Dimensionen nach Fend (2006).

Die Dimension Integrationskraft greift die rein systemische Integration von Kindern und Jugendlichen in das Schulsystem auf. Darüber hinaus zählt hier auch die soziale Integration mit rein, d.h. dass Kindern über die Partizipation an besonderen Lernsettings zusätzliche Gelegenheiten der sozialen Begegnung mit anderen sowie Teilhabe an kollektiven Lernprozessen ermöglicht werden, beispielsweise über Bildungs-, Betreuungs- und Freizeitangebote in den Bildungslandschaften.

Die Durchlässigkeit bezieht sich auf die Allokationsfunktion von Schulsystemen und fokussiert die Frage, inwiefern sich Selektionsprozesse unabhängig von individuellen sozioökonomischen Merkmalen vollziehen. In Anlehnung an Manitius (2013) finden durchlässigkeitsbefördernde Bildungslandschaftsaktivitäten insbesondere im Hinblick auf die Gestaltung eines gelingenden Übergangs zwischen den Bildungsinstitutionen (z. B. Kindergarten - Schule) statt.

Die Dimension der Kompetenzförderung zielt auf die Fähigkeit des Schulsystems ab, dass Kinder und Jugendliche unabhängig von ihrer sozioökonomischen Lage, ihres Geschlechts oder ihrer Hautfarbe vergleichbare Lernbedingungen vorfinden und auch zu vergleichbaren Lernergebnissen kommen. Eine gerechte Kompetenzförderung fördert die Potenziale aller Kinder und Jugendlichen. Berkemeyer et al. (2017) fordern hierbei auch ein Mindestmaß an kognitiven und moralischen Kompetenzen.

Die Zertifikatsvergabe hängt mit der Kompetenzförderung zusammen und betrachtet die Anforderungen sowie die Vergleichbarkeit von Bildungszertifikaten über Landesgrenzen hinweg.

Die Konzeption des Chancenspiegels konkretisiert den abstrakten Begriff der Chancengerechtigkeit und macht ihn somit messbar, weshalb er als Grundlage für die vorliegende Untersuchung gewählt wurde. Auch wenn sich die Ausführungen des Chancenspiegels auf die Analyse von Chancengerechtigkeit im Schulsystem be-

\footnotetext{
1 Entgegen der Interpretation Giesingers (2015) stellen Berkemeyer et al. (2017) klar, dass es hier kein hierarchisches Verhältnis zwischen den Kernaspekten der Teilhabe, der fairen Institution und der Anerkennung gibt. Vielmehr sehen die Autoren und Autorinnen des Chancenspiegels im Aspekt der Teilhabe ein die drei Gerechtigkeitsvorstellungen verbindendes Element.
} 
ziehen, haben Manitius et al. (2015) bereits bewiesen, dass sich diese Dimensionen auch auf die Analyse von gerechtigkeitsbezogenen Unterstützungsleistungen durch Regionalisierungsmaßnahmen für das Schulsystem anwenden lassen. Allerdings beschränken sich ihre Ausführungen hauptsächlich auf die Kooperation verschiedener Bildungsinstitutionen. Aus diesem Grund greift die vorliegende Analyse auf die Beschreibungen von Manitius et al. (2015) zu gerechtigkeitsbezogenen Unterstützungsleistungen von Regionalisierung bezogen auf die oben erläuterten Gerechtigkeitsdimensionen des Schulsystems zurück und erweitert sie um gerechtigkeitsfördernde Aspekte für die Bildungsabnehmenden, also für die Kinder, Jugendlichen und Eltern, in dem auch die in Bildungslandschaften entstandenen Freizeit-, Bildungs- und Betreuungsangebote in die Betrachtung mit einfließen. In einem nächsten Schritt sollen diese empirisch untersucht und so aufgezeigt werden, welche Gerechtigkeitsdimensionen mit den einzelnen Bildungsangeboten (z. B. Sprachkurse) und auch die Netzwerkaktivitäten berücksichtigt werden.

\section{Methoden}

Die Untersuchung ist angelegt im Programm „Bildungslandschaften Schweiz“, welches 2014 von der Jacobs Foundation als Public Private Partnership mit staatlichen Partnern initiiert wurde. Das Programm hat sich als übergeordnetes Ziel gesetzt, Kindern und Jugendlichen gerechtere Chancen auf eine qualitativ gute und umfassende Bildung zu ermöglichen (Jacobs Foundation 2011). Dies wird angestrebt durch den Aufbau von Bildungslandschaften. Bildungslandschaft ist definiert als eine gezielte und systematische, an pädagogischen Zielgrössen orientierte institutionalisierte Zusammenarbeit von Bildungsakteuren im Mehrebenensystem Bildung auf kantonaler, regionaler und kommunaler Ebene, bestehend aus Institutionen und Personen aus dem schulischen und außerschulischen Bereich, mit dem Ziel, die Quantität und Qualität von Bildungsangeboten für Kinder und Jugendliche zu steigern (Ebd.). Im Gegensatz zu deutschen Bildungslandschaften unterscheiden sich die Bildungslandschaften aus diesem Programm also darin, dass sie zusätzlich zur lokalen Koordination auch auf regionaler Ebene koordiniert werden. Dieses Programm erlaubt Feldzugang für eine auf fünf Jahre angelegte Forschungsstudie. Während der Programmlaufzeit sind insgesamt 22 Bildungslandschaften in der deutsch- und französischsprachigen Schweiz entstanden. Die Interventionen des Programms bestehen zum einen in der Bereitstellung finanzieller Ressourcen und zum anderen in der Bereitstellung einer externen Prozessbegleitung.

Auch wenn sich alle Bildungslandschaften inhaltlich an den Programmzielen orientiert haben, unterscheiden sie sich in Bezug auf ihre Ausgangslage, ihren Aufbau, ihre Vorgehensweise sowie ihrer konkreten Ausgestaltung. In drei Fällen entsprachen die entstandenen Bildungslandschaften jedoch nicht den von uns formulierten Kriterien einer Bildungslandschaft. Um eine bessere Vergleichbarkeit zu gewährleisten, wurden aus diesem Grund also die Aktivitäten während der Projektlaufzeit von 19 Bildungslandschaften in die Betrachtungen mit aufgenommen.

Die hier vorgestellte explorative und qualitative Teiluntersuchung ist querschnittlich angelegt und betrachtet basierend auf Dokumenten, die im Rahmen der Pro- 
grammlaufzeit entstanden sind, die Maßnahmen, mit denen innerhalb der Bildungslandschaften Chancengerechtigkeit gefördert werden soll.

\subsection{Dokumente}

Bei dem Datenmaterial handelt es sich um Dokumente. Die Dokumentenanalyse umfasst ,,sämtliche gegenständliche Zeugnisse, die als Quelle zur Erklärung menschlichen Verhaltens dienen können“ (Atteslander 1971, S. 53). In der vorliegenden Untersuchung soll die Dokumentenanalyse genutzt werden, um die im Rahmen von Bildungslandschaften entstandenen Maßnahmen und Aktivitäten in Bildungslandschaften auszuwerten. Die Dokumente aus den Bildungslandschaften offenbaren hierbei ,institutionalisierte Spuren, d.h., dass aus ihnen legitime Schlussfolgerungen über Aktivitäten, Absicht und ihrer Verfasser bzw. der von ihnen repräsentierten Organisationen gezogen werden können“ (Wolff 2000, S. 503).

Während der Projektlaufzeit wurden diverse Dokumentationen und Reportings u. Ä. über die Entwicklung und Aktivitäten der Bildungslandschaften von den Projektleitungen angefertigt, aus denen unter anderem hervorgeht, welche Bildungs-, Betreuungs- und Freizeitangebote für die Bevölkerung, also für Kinder, Jugendliche und/oder Eltern und welche Netzwerkaktivitäten für die Bildungsakteure, beispielsweise Lehrkräfte, Mitarbeitende aus Jugendzentren, Vereinen oder Bibliotheken, entstanden sind.

Die Daten wurden während der gesamten Förderlaufzeit, also über einen Zeitraum von vier Jahren, gesammelt. Insgesamt wurden 178 Dokumente (z. B. Websites, Reportings, Dokumentationen und Broschüren) von 19 Bildungslandschaften aus der deutsch- und französischsprachigen Schweiz berücksichtigt, aus denen 190 Aktivitäten identifiziert werden konnten. Die Anzahl der Aktivitäten pro Bildungslandschaft variiert zwischen 4 und 19.

Zur Untersuchung wurden folgenden Materialien zur Beantwortung der forschungsleitenden Fragen herangezogen:

- Projektreportings, die von den Bildungslandschaften während der Projektlaufzeit angefertigt wurden $(n=73)$

- Schluss- und Transferberichte der Bildungslandschaften $(n=19)$

- Informationen zu Aktivitäten innerhalb der Bildungslandschaften auf den jeweiligen Websites $(n=19)$, aus Flyern, (Zwischen-)Evaluationen oder anderen Dokumentationen $(n=67)$

Während die Projektreportings sowie die Schluss- und Transferberichte als Berichtslegungsgrundlage an die geldgebende Stiftung dienten und einer einheitlichen Gliederung unterliegen, sind die Informationen zu den Aktivitäten innerhalb der Bildungslandschaften auf Websites, Flyern oder anderen Dokumentationen auf Initiative vereinzelter Bildungslandschaften entstanden, d. h. sie unterscheiden sich zum einen in den Aspekten zu den einzelnen Aktivitäten und sind auch nicht für jede Bildungslandschaft verfügbar. Folglich variiert auch der Umfang an Informationen zu den Aktivitäten innerhalb der 19 Bildungslandschaften. Die Dokumente unterscheiden sich zwar im Detaillierungsgrad, wurden in der Auswertung aber gleich gewichtet. 
Für die Analyse wurden nur die Maßnahmen und Angebote berücksichtigt, die im Rahmen des Programms „Bildungslandschaften Schweiz“ und somit folglich unter der Zielsetzung, die Chancengerechtigkeit zu erhöhen, entstanden sind. Es können keine Aussagen dazu getroffen werden, ob die Bildungslandschaftsaktivitäten von den Empfängern und Empfängerinnen - also den Bildungsakteuren und der Bevölkerung - als unterstützend wahrgenommen werden oder ob diese dann auch tatsächlich eine Wirkung entfalten. Es handelt sich vielmehr um die Bereitstellung dieser Aktivitäten innerhalb der Bildungslandschaften.

\subsection{Qualitative Inhaltsanalyse}

Da die Daten theoriegeleitet analysiert werden sollen, wurden die Dokumente mithilfe einer strukturierenden qualitativen Inhaltsanalyse ausgewertet (Mayring 2002 2012). Die Hauptkategorien des Kategoriensystems wurden deduktiv hergeleitet und basieren zum einen auf den Ausführungen des Chancenspiegels. Darüber hinaus basiert ein Teil der Subkategorien auf den Überlegungen von Manitius et al. (2015). Zum anderen wurden bei der Kodierung der Bildungslandschaftsaktivitäten auch induktiv Subkategorien gebildet (Mayring 2008).

Durch das Erstellen einer Kodieranleitung mit Kategoriendefinitionen sowie Ankerbeispielen wurden zudem die Sicherstellung von Objektivität und intersubjektiver Nachvollziehbarkeit gewährleistet (Ebd.). In einem nächsten Schritt wurden die Dokumente nach ihrer Relevanz bezüglich der Bildungslandschaftsaktivitäten während der Projektlaufzeit untersucht. Diese wurden in einer Übersicht tabellarisch mit einer Beschreibung zu Inhalt, Zielen und Zielgruppen erfasst. Anschließend wurden die Namen der Aktivitäten basierend auf der Beschreibung mithilfe von MAXQDA kodiert.

Bei der Dimension Integrationskraft sollen Möglichkeitsräume sozialer Integration von Kindern und Jugendlichen sowie Eltern beleuchtet werden. Hierzu können beispielsweise Begegnungsorte wie Spielplätze und Elterncafés zählen.

Bildungslandschaftsaktivitäten, die der Förderung der Durchlässigkeit des Schulsystems zugeordnet werden sollen, umfassen zum einen die Stärkung der Kooperation zwischen den Bildungsinstitutionen, die an Übergängen beteiligt sind, und zum anderen die Schaffung von Maßnahmen für Kinder und Eltern, die den Übergang erleichtern sollen. Manitius et al. (2015) denken hier zum Beispiel an Beratungsangebote.

Die Schule gilt als primärer Ort, in dem Kinder und Jugendliche Kompetenzen erwerben. In den letzten Jahren gewinnen jedoch non-formale und informelle Lernsettings immer mehr an Bedeutung. Auch in Bildungslandschaften spielt der Kompetenzerwerb in außerschulischen Lernorten eine Rolle. Folglich können Bildungslandschaften bezogen auf Kompetenzförderung eine Unterstützungsleistung erbringen, indem sie kompetenzvermittelnde Bildungsangebote (z. B. Sprachförderung) für Kinder oder Eltern initiieren bzw. ihren Ausbau fördern. Gleichzeitig können Bildungslandschaften auch eine Kompetenzförderung von Bildungsinstitutionen unterstützen, indem wechselseitige Lernprozesse zwischen Bildungsakteuren ermöglicht werden, z. B. in Form von (institutionenübergreifenden) Weiterbildungsangeboten (Manitius 2013). 
Tab. 1 Übersicht über Haupt- und Subkategorien und über die jeweiligen Zielgruppen

\begin{tabular}{|c|c|c|}
\hline $\begin{array}{l}\text { Zu unterstützende Gerechtig- } \\
\text { keitsdimension d. Schulsys- } \\
\text { tems (Hauptkategorie) }\end{array}$ & Zielgruppe & $\begin{array}{l}\text { Beispiele der Leistungen von Bildungslandschaften } \\
\text { (Subkategorie) }\end{array}$ \\
\hline \multirow[t]{3}{*}{ Integrationskraft } & Akteure & $\begin{array}{l}\text { Netzwerkbildung, um institutionelle Grenzen zu } \\
\text { überwinden }\end{array}$ \\
\hline & Bevölkerung & Schaffung von Austausch- und Begegnungsorten \\
\hline & & Informierende Angebote \\
\hline \multirow[t]{2}{*}{ Durchlässigkeit } & Akteure & Anregung professioneller Kooperation \\
\hline & Bevölkerung & Schaffung adäquater Beratungsangebote \\
\hline \multirow[t]{2}{*}{ Kompetenzförderung } & Akteure & Fortbildungsmanagement \\
\hline & Bevölkerung & Kompetenzfördernde Angebote \\
\hline Zertifikatsvergabe & Bevölkerung & $\begin{array}{l}\text { Anschlussmöglichkeiten v. a. für Abgänger ohne } \\
\text { Abschluss sowie adäquate Beratungsangebote für } \\
\text { die Betroffenen }\end{array}$ \\
\hline
\end{tabular}

Da die Zertifikatsvergabe überwiegend in der Schule stattfindet, ist es nicht möglich, die Aktivitäten innerhalb der Bildungslandschaften als Unterstützungsleistung der Zertifikatsvergabe an Schulen einzuordnen. Stattdessen ist es jedoch denkbar, dass eine nicht-gelungene Zertifikatsvergabe durch Aktivitäten der Bildungslandschaften kompensiert werden könnte. Hierunter würden beispielsweise Maßnahmen zur erfolgreichen Integration von Schulabgängerinnen und Schulabgängern ohne Schulabschluss in den Arbeitsmarkt zählen (Manitius et al. 2015) (Tab. 1).

Das Vorgehen, den Kodierleitfaden sowie die Kategoriendefinitionen wurden regelmäßig im Projektteam diskutiert, um eine interne Validität sicher zu stellen. Das Kategoriensystem wurden zudem bezüglich Intra- und Intercoderübereinstimung anhand der Berechnung von Cohens Kappa nach Brennan und Prediger (1981) überprüft. Zunächst hat ein Kodierer dasselbe Sample zweimal im Abstand von zwei Wochen kodiert. Die Überprüfung der Intrakoderreliabität ergab einen Reliabilitätskoeffizienten von $R=0,81$, was gemäss Landis und Koch (1977) einem fast perfekten Wert entspricht.

Die Interkoderreliabilität wurde anhand von 5 zufällig ausgewählten der insgesamt 19 Übersichten geprüft. Anhand der Kappa-Formel ergab diese einen Reliabilitätskoeffizienten von $R=0,76$, der als substanziell bewertet werden kann (ebd.).

\section{Ergebnisse}

Das Ziel der vorliegenden Dokumentenanalyse besteht darin, empirische Hinweise über die Leistungen von Bildungslandschaften zu erhalten, auf welche Gerechtigkeitsdimensionen sich diese beziehen sowie für welche Zielgruppen diese Unterstützungsleistungen erbracht werden und mit welchen Zielen sie verknüpft werden.

Im Rahmen der Dokumentenanalyse konnten verschiedene während der Förderlaufzeit neu entstandene Maßnahmen innerhalb der Bildungslandschaften aufgedeckt werden, die auf eine Erhöhung der Chancengerechtigkeit abzielen. Insgesamt wur- 


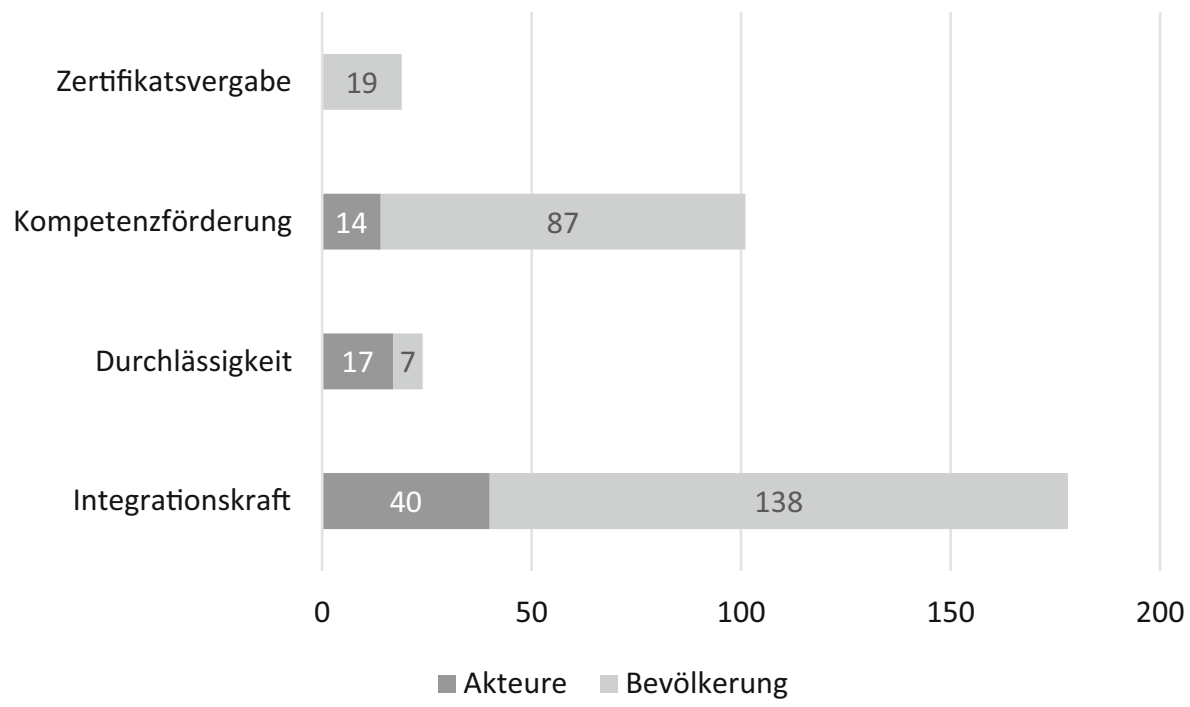

Abb. 1 Anzahl der Kodierungen der Unterstützungsleistungen. (Eigene Darstellung)

den 322 Kodierungen vergeben. Eine Übersicht über die kodierten Unterstützungsleistungen sowie deren Häufigkeiten findet sich in Abb. 1.

In der Abbildung werden die Hauptkategorien des Chancenspiegels, die jeweils noch einmal in Subkategorien unterschieden werden, dargestellt, um die vielfältigen Aktivitäten der Bildungslandschaften zu systematisieren und beschreibbar zu machen. Es wird weiterhin unterschieden, ob sich die kodierten Maßnahmen an die Bevölkerung oder an die Bildungsakteure richten.

\subsection{Verortung des gerechtigkeitsfördernden Potenzials von Bildungslandschaften auf der Dimension der Integrationskraft}

Betrachtet man die einzelnen Unterstützungsdimensionen, fällt auf, dass etwas mehr als die Hälfte $(n=178)$ der vergebenen Kodierungen der Dimension Integrationskraft zugewiesen werden. Wenn man also basierend auf der Anzahl der vergebenen Kodierungen die Bedeutsamkeit der Dimensionen ableiten möchte, dann lässt sich das gerechtigkeitsfördernde Potenzial von Bildungslandschaften auf der Dimension Integrationskraft verorten. In allen betrachteten Bildungslandschaften konnten Kodierungen dieser Dimension vergeben werden. Die überwiegende Mehrheit der Aktivitäten dieser Dimension richtet sich dabei an die Bevölkerung. Insgesamt entfallen 96 Kodierungen auf die Subkategorie Schaffung von Austausch- und Begegnungsgelegenheiten für die Bevölkerung, 42 Kodierungen auf die der informierenden Angebote für die Bevölkerung und 40 auf die Netzwerkbildung zur Überwindung institutioneller Grenzen. In der Subkategorie Schaffung von Austausch- und Begegnungsgelegenheiten finden sich insbesondere gruppenbezogene Aktivitäten wie Quartierfeste, diverse Treffs für Eltern und/oder Kinder sowie Angebote zur Bewegungsförderung. Die Zielsetzung dieser Angebote besteht im Allgemeinen darin, 
Gelegenheiten zu schaffen, bei denen sich die Eltern und/oder Kinder bzw. Jugendliche begegnen und informell austauschen können. So soll einer Exklusion von Bevölkerungsgruppen entgegengewirkt werden., wie die folgenden Zitate exemplarisch verdeutlichen:

Das Elterncafé bietet wöchentlich einen Ort der Begegnung und des Austausches zwischen Eltern, Lehrpersonen und dem Quartier.

Es [Angebot für Bewegungsförderung] fördert die Freude an Bewegung und gibt die Möglichkeit, neue Freundschaften zu knüpfen.

Bei den informierenden Angeboten - der zweiten Subkategorie der Dimension Integrationskraft - handelt es sich im Wesentlichen um an die Bevölkerung gerichtete Maßnahmen, deren Hauptziele darin bestehen, dass sie entweder über bereits bestehende Bildungsangebote in der Gemeinde oder über das Bildungssystem informieren, um so die Voraussetzung zur Nutzung eines entsprechenden Bildungsangebots bzw. -gangs zu schaffen. Ein Großteil dieser Angebote findet punktuell, d.h. einmal im Jahr bzw. einmal alle sechs Monate, statt. Sofern Maßnahmen dieser Dimension über bestehende Angebote informieren, sind sie von Kommunikationsmaßnahmen abzugrenzen, da sie den Individuen in der Regel die Möglichkeit geben, einen vertieften Eindruck dieser Angebote zu erhalten, z. B. in dem sie diese selbst ausprobieren können oder mit den durchführenden Akteuren ins Gespräch kommen können und sich so mit gezielten Fragen ein genaues Bild über das entsprechende Angebot machen können. Mitunter handelt es sich hier auch um Beratungsangebote, die das Angebot gezielt empfehlen oder im Rahmen dessen die Eltern bzw. Kinder beim ersten Besuch des entsprechenden Angebots begleitet werden. Beide Aspekte dienen dazu, dass Hemmschwellen abgebaut werden und so Bevölkerungsgruppen, die sich seltener an zusätzlichen Bildungsangeboten beteiligen, auf diese Weise ermutigt werden sollen, das Angebot in Anspruch zu nehmen. Allen 19 Bildungslandschaften ist zudem gemein, dass sie Kommunikationsmaßnahmen in Form von Homepages, Flyern, Broschüren etc. zur Sichtbarmachung der Bildungsangebote in der Gemeinde durchgeführt haben.

Darüber hinaus wurden in der Kategorie Integrationskraft auch Maßnahmen zur Netzwerkbildung ausgemacht, die sich an die Akteure der verschiedenen Bildungsinstitutionen richten. Hierunter fallen zum Beispiel Netzwerktreffen und Austauschplattformen, die den Austausch und die Zusammenarbeit der Bildungsakteure verschiedener Bildungsstufen und Bildungsinstitutionen forcieren soll. Aus den Dokumenten geht hervor, dass diese Aktivitäten darauf abzielen, ,eine gemeinsame Verantwortung für die Bildungsbiografie der Kinder und Jugendlichen im Quartier“" zu fördern und zu verstärken. Zudem dienen diese Aktivitäten auch dazu, dass die Bildungsakteure voneinander wissen und so auch die Kinder, Jugendlichen und/oder Eltern an die entsprechenden Angebote verweisen können.

\subsection{Kodierungen, die der Dimension Kompetenzförderung zugeordnet wurden}

Die zweitmeisten Kodierungen $(n=101)$ entfallen auf die Dimension Kompetenzförderung, die Maßnahmen beinhaltet, die eine Steigerung verschiedener Kompetenzen entweder für die Bevölkerung (z.B. Sprachförderung) oder der Bildungsakteure 
(z.B. Fortbildung) beinhalten. Während 17 Bildungslandschaften von Aktivitäten berichten, die auf Kinder, Jugendliche und Eltern abzielen, konnten in 10 Bildungslandschaften Maßnahmen für Akteure ausgemacht werden. In zwei Bildungslandschaften konnten keine Aktivitäten dieser Dimension zugeordnet werden.

Betrachtet man die Anzahl der Maßnahmen, die sich an die Akteure und an die Bevölkerung richten, dann fällt sofort auf, dass kompetenzfördernde Maßnahmen für Bildungsakteure (z. B. Fortbildungsmaßnahmen) wesentlich seltener geschaffen wurden als kompetenzfördernde Maßnahmen für die Bevölkerung (s. Abb. 1). Die Kompetenzen, die im Rahmen dieser Angebote gefördert werde sollen, sind äußerst vielfältig. Bei den kompetenzfördernden Angeboten, die sich an Eltern richten, stehen hauptsächlich die Vermittlung erzieherischer Kompetenzen, das Erlernen der deutschen Sprache und Elterncoaching-Maßnahmen zur Auswahl. Unter kompetenzfördernde Maßnahmen für Kinder und Jugendliche fallen neben Sprachförderangeboten unter anderem auch Maßnahmen, die die Motorik und Sozialkompetenz fördern sollen. Bei den Akteuren stehen gemeinsame Fortbildungen im Vordergrund.

\subsection{Kodierungen, die den Dimensionen Durchlässigkeit und Zertifikatsvergabe zugeordnet wurden}

Auf die Dimensionen Durchlässigkeit und Zertifikatsvergabe, die miteinander zusammenhängen, entfallen insgesamt 43 Kodierungen. Kodierungen auf der Dimension Durchlässigkeit entfallen auf Maßnahmen, die auf eine Verbesserung des Übergangs zwischen Bildungsinstitutionen abzielen. Alle 19 Bildungslandschaften haben zu Projektbeginn einen Übergang festgelegt, den sie im Rahmen ihrer Bildungslandschaft fokussieren wollen. 14 Bildungslandschaften haben den Übergang vom Frühbereich in den Kindergarten und 4 Bildungslandschaften den Übergang Sekundarstufe I zu Berufsbildung ausgewählt; eine Bildungslandschaft hat sowohl den Übergang vom Frühbereich in den Kindergarten sowie den Übergang von der Primar- zu Sekundarstufe I benannt. Bei dieser Dimension konnten mehr Aktivitäten auf der Ebene der Akteure $(n=9)$ als auf der Ebene der Bevölkerung $(n=6)$ zugeordnet werden. Obwohl zwar alle Bildungslandschaften zu Projektbeginn einen Übergang festgelegt haben, den sie verbessern möchten, konnten in 8 Bildungslandschaften keine Aktivitäten auf der Dimension Durchlässigkeit ausgemacht werden.

Mit der Subkategorie Anregung professioneller Kooperation ist eine Vernetzungsaktivität zwischen auf- und abnehmenden Bildungsinstitutionen, bspw. eine Vernetzung zwischen Frühbereich und Kindergarten oder gemeinsame Fortbildungsaktivitäten, die sich an Akteure aus zu- und abnehmende Bildungsinstitutionen richten, gemeint.

Kodierungen auf der Ebene Zertifikatsvergabe beinhalten Maßnahmen, die Anschlussmöglichkeiten nach der Schule, besonders für Jugendliche, die voraussichtlich keinen Abschluss machen werden, aufzeigen.

Diese Dimension wird bereits indirekt durch die Aktivitäten auf der Dimension der Durchlässigkeit mit abgedeckt. In den Bildungslandschaften, die am Übergang von der Sekundarstufe I zur Berufsbildung arbeiten, können jedoch auch Aktivitäten ausgemacht werden, die direkt auf dieser Dimension angesiedelt werden können $(n=4)$. Die hier zugeordneten Aktivitäten richten sich ausschließlich an Jugendliche 
und konzentrieren sich darauf, die Jugendlichen in ihrem Berufswahlprozess - sei es durch die Wahl eines Berufs oder durch das Schreiben eines Bewerbungsschreibens oder das Führen eines Bewerbungsgesprächs - zu unterstützen. Vereinzelte Maßnahmen wurden auch speziell für die Jugendlichen geschaffen, die vermutlich Probleme bei diesem Übergang haben werden (z. B. durch einen fehlenden Schulabschluss). Auffällig ist, dass hier von keinen Maßnahmen für Eltern berichtet wird, obschon aus dem bisherigen Stand der Forschung hervorgeht, dass diese im Berufswahlprozess des Jugendlichen eine bedeutende Rolle einnehmen (Kracke und Noack 2006).

\section{Diskussion}

Es zeigt sich, dass sowohl die Netzwerkaktivitäten als auch die im Rahmen der Projektlaufzeit geschaffenen Freizeit-, Betreuungs- sowie Bildungsangebote in den Bildungslandschaften verschiedene Gerechtigkeitsdimensionen berücksichtigen. Im Gegensatz zur Arbeit von Manitius et al. (2015), die in ihren Analysen zu dem Ergebnis kommen, dass die Aktivitäten von Bildungsbüros insbesondere auf die Unterstützung der Durchlässigkeit von Schulsystemen abzielen, verdeutlichen die Befunde dieses Beitrags, dass die Aktivitäten der hier betrachteten Bildungslandschaften vor allem Aspekte der Integrationskraft berühren. Hierzu zählen auf Ebene der Bevölkerung die Schaffung von Austausch- und Begegnungsgelegenheiten und informierenden Angebote sowie die Sichtbarmachung vorhandener Angebote durch Websites, Flyer u. ä. und auf Ebene der Akteure netzwerksbildungsfördernde Maßnahmen. Da in allen Bildungslandschaften Bemühungen zur Sichtbarmachung und größeren Bekanntheit bestehender Bildungsangebote ausgemacht werden können, könnten alle hier betrachteten Bildungslandschaften einen potenziellen Beitrag auf der Dimension der Integrationskraft leisten und so die Chancen der Kinder und Jugendlichen sowie Eltern erhöhen, ein Angebot wahrzunehmen. Dieser Befund ist insofern wenig überraschend, da anzunehmen ist, dass Aktivitäten wie die Bekanntmachung des bestehenden Bildungsangebots sowie die Vernetzung unter den Bildungsakteuren erste Schritte beim Aufbau einer Bildungslandschaft darstellen, bevor eine Bearbeitung der weiteren Dimensionen erfolgt.

Doch auch die anderen Gerechtigkeitsdimensionen wurden durch die Aktivitäten in den Bildungslandschaften berücksichtigt, wenn auch nicht im gleichen Umfang wie die Dimension der Integrationskraft. Es zeigt sich ebenfalls, dass die Aktivitäten in keiner Bildungslandschaft allen vier Dimensionen zugeordnet werden konnten. Vielmehr decken die Aktivitäten in den meisten Bildungslandschaften zwei bis drei Dimensionen des Chancenspiegels ab. Die Befunde verdeutlichen ebenfalls, dass die Mehrheit der Aktivitäten auf die Bevölkerung abzielt. Hier stehen besonders die Schaffung von integrativen sowie kompetenzfördernden Maßnahmen im Vordergrund. Kompetenzförderung von Bildungsakteuren kommt eher eine nachgeordnete Rolle zu. Dies wird an der geringeren Anzahl der Kodierungen in dieser Kategorie deutlich.

Um eine umfassende und ganzheitliche Bildung der Kinder und Jugendlichen zu gewährleisten, sollte die Angebotspalette in den Bildungslandschaften möglichst viele Gerechtigkeitsdimensionen abdecken. Es ist also die Aufgabe der Bildungsakteure 
in der Praxis zu reflektieren, inwiefern die Aktivitäten in den Bildungslandschaften diese Gerechtigkeitsdimensionen abdecken.

Auch wenn in dieser Analyse empirische Hinweise darüber gewonnen werden konnten, welche Gerechtigkeitsdimensionen mit den einzelnen Aktivitäten in den Bildungslandschaften berücksichtigt werden, können keine Aussagen dazu getroffen werden, ob die Bildungslandschaftsaktivitäten von den Empfängern und Empfängerinnen als unterstützend wahrgenommen werden oder ob diese dann auch tatsächlich eine Wirkung entfalten - hierzu bedarf es weiterführender Analysen. Der vorliegende Beitrag fokussiert vielmehr die Bereitstellung gerechtigkeitsfördernder Aktivitäten innerhalb der Bildungslandschaften. Zudem basiert die Analyse auf Aktivitäten von denen in den Bildungslandschaftsdokumenten berichtet wird. Falls die Auflistung der Maßnahmen der Bildungslandschaften in den Dokumenten nicht vollständig sein sollte, sind diese Maßnahmen in die vorliegende Betrachtung also auch nicht miteingeflossen. Schließlich betrachtet dieser Beitrag lediglich die ersten fünf Jahre einer Entwicklung der Bildungslandschaft. Weitere Analysen wären nötig, um zu untersuchen, ob die Bearbeitung weiterer Gerechtigkeitsdimensionen möglicherweise erst in den Fokus der Akteure tritt, beispielsweise nach dem andere Aktivitäten vorgängig durchgeführt wurden.

Danksagung An dieser Stelle möchte ich der Pädagogischen Hochschule Zug meinen großen Dank aussprechen, die mich bei der Anfertigung dieses Beitrags unterstützt hat.

Funding Open access funding provided by University of Teacher Education Zug

Open Access Dieser Artikel wird unter der Creative Commons Namensnennung 4.0 International Lizenz veröffentlicht, welche die Nutzung, Vervielfältigung, Bearbeitung, Verbreitung und Wiedergabe in jeglichem Medium und Format erlaubt, sofern Sie den/die ursprünglichen Autor(en) und die Quelle ordnungsgemäß nennen, einen Link zur Creative Commons Lizenz beifügen und angeben, ob Änderungen vorgenommen wurden.

Die in diesem Artikel enthaltenen Bilder und sonstiges Drittmaterial unterliegen ebenfalls der genannten Creative Commons Lizenz, sofern sich aus der Abbildungslegende nichts anderes ergibt. Sofern das betreffende Material nicht unter der genannten Creative Commons Lizenz steht und die betreffende Handlung nicht nach gesetzlichen Vorschriften erlaubt ist, ist für die oben aufgeführten Weiterverwendungen des Materials die Einwilligung des jeweiligen Rechteinhabers einzuholen.

Weitere Details zur Lizenz entnehmen Sie bitte der Lizenzinformation auf http://creativecommons.org/ licenses/by/4.0/deed.de.

\section{Literatur}

Atteslander, P. (1971). Methoden der Empirischen Sozialforschung. New York: Walter de Gruyter.

Bauer, P., Bolay, E., Gschwind, A. K., \& Zipperle, M. (2017). MABEV - Metaanalyse, Bestandsaufnahme \& Evaluation des Auf- und Ausbaus Lokaler Bildungsnetze (LoBiN). Eine Expertise im Rahmen des „Zukunftsplan Jugend“. https://sozialministerium.baden-wuerttemberg.de/fileadmin/redaktion/msm/intern/downloads/Publikationen/ZPJ_MABEV_Gesamtbericht_2017_Internet.pdf. Zugegriffen: 01. Mai 2019

Becker, R. (Hrsg.). (2009). Lehrbuch der Bildungssoziologie (1. Aufl.). Wiesbaden: VS.

Bell, D. (1999). The coming of post-industrial society: a venture in social forecasting. New York: Basic Books. Special anniversary 
Berkemeyer, N., Järvinen, H., \& Mauthe, A. (2009). Schulen im Team. Kommunales Management von Schulnetzwerken. In N. Berkemeyer, H. Kuper, V. Manitius \& K. Müthing (Hrsg.), Schulische Vernetzung. Eine Übersicht zu aktuellen Netzwerkprojekten (S. 171-188). Münster: Waxmann.

Berkemeyer, N., Bos, W., Manitius, V., \& Strietholt, R. (2012). Chancenspiegel: Zur Chancengerechtigkeit und Leistungsfähigkeit der deutschen Schulsysteme (2. Aufl.). Gütersloh: Bertelsmann.

Berkemeyer, N., Bos, W., Hermstein, B., Abendroth, S., \& Semper, I. (2017). Chancenspiegel - eine Zwischenbilanz: Zur Chancengerechtigkeit und Leistungsfähigkeit der deutschen Schulsysteme seit 2002. Gütersloh: Bertelsmann.

Berse, C. (2009). Mehrdimensionale Bildung im Kontext kommunaler Bildungslandschaften: Bestandsaufnahme und Perspektiven. Opladen: Budrich UniPress.

BJK, (Bundesjugendkuratorium) (2002). Bildung ist mehr als Schule. Leipziger Thesen zur aktuellen bildungspolitischen Debatte. Gemeinsame Erklärung des Bundesjugendkuratoriums, der Sachverständigenkommission des 11. Kinder- und Jugendberichts und der Arbeitsgemeinschaft für Jugendhilfe. Bonn / Berlin / Leipzig: Eigenverlag.

BMFSFJ, (Bundesministerium für Familie, Senioren, Frauen und Jugend) (2005). 12. Kinder- und Jugendbericht. Bericht über die Lebenssituation junger Menschen und die Leistungen der Kinder- und Jugendhilfe in Deutschland. Eigenverlag. https://www.bmfsfj.de/blob/112224/ 7376e6055bbcaf822ec30fc6ff72b287/12-kinder-und-jugendbericht-data.pdf. Zugegriffen: 14. Okt. 2017

Brennan, R.L., \& Prediger, D.J. (1981). Coefficient Kappa: Some Uses, Misuses, and Alternatives. Educational and Psychological Measurement, 41(3), 687-699

Duveneck, A. (2016). Bildungslandschaften verstehen: Zum Einfluss von Wettbewerbsbedingungen auf die Praxis (1. Aufl.). Weinheim / Basel: Beltz Juventa.

Fend, H. (2006). Neue Theorie der Schule: Einführung in das Verstehen von Bildungssystemen (1. Aufl.). Wiesbaden: VS.

Fend, H., Berger, F., \& Grob, U. (2004). Langzeitwirkungen von Bildungserfahrungen am Beispiel von Lesen und Computer Literacy. Ergebnisse der LIFE-Studie (Lebensverläufe von der späten Kindheit ins frühe Erwachsenenalter). Zeitschrift für Pädagogik, 50(1), 56-76.

Giesinger, J. (2015). Bildungsgerechtigkeit und die sozialen Funktionen der Schule. Zu den normativen Grundlagen des Chancenspiegels. In V. Manitius, B. Hermstein, N. Berkemeyer \& W. Bos (Hrsg.), Zur Gerechtigkeit von Schule. Theorien, Konzepte, Analysen (S. 150-163). Münster: Waxmann.

Honneth, A. (2011). Das Recht der Freiheit: Grundriss einer demokratischen Sittlichkeit (1. Aufl.). Berlin: Suhrkamp.

Hradil, S. (2006). Die Sozialstruktur Deutschlands im internationalen Vergleich (2. Aufl.). Wiesbaden: VS.

Huber, S. G. (2014). Kooperation in Bildungslandschaften: Aktuelle Diskussionsstränge, Wirkungen und Gelingensbedingungen. In S. G. Huber (Hrsg.), Kooperative Bildungslandschaften. Netzwerke(n) in und mit System (S. 3-29). Köln: WoltersKluwer.

Huber, S. G., Schwander, M., Kilic, S., \& Wolfgramm, C. (2014). Bildungslandschaften - Übersicht über exemplarische Projekte und Evaluationen. In S. G. Huber (Hrsg.), Kooperative Bildungslandschaften. Netzwerke(n) in und mit System (S. 137-164). Köln: WoltersKluwer.

Huber, S. G., Werner, R., Koszuta, A., Schwander, M., Strietholt, R., Bacso, M.-A., Gürel, E., Schneider, J., Hürlimann, F., \& Nonnenmacher, L. (2019). Wissenschaftliche Evaluation und Erforschung des Programms „Bildungslandschaften Schweiz“. Abschlussbericht. Unveröffentlichtes Dokument. Institut für Bildungsmanagement und Bildungsökonomie, IBB.

Jacobs Foundation (Hrsg.). (2011). Bildungslandschaften Schweiz - Umfassende Bildungsqualität gemeinsam entwickeln. http://bildungslandschaften.ch/sites/default/files/Konzept\%20Bildungslandschaften \%20Schweiz\%20JF.pdf. Zugegriffen: 04. März 2014

Järvinen, H., Sendzik, N., Sartory, K., \& Otto, J. (2015). Unterstützungssysteme im Kontext von Regionalisierungsprozessen. Eine theoretische und empirische Annäherung. Journal for educational research online, 7(4), 94-124.

Jungermann, A., Manitius, V., \& Berkemeyer, N. (2015). Regionalisierung im schulischen Kontext. Ein Überblick zu Projekten und Forschungsbefunden. Journal for educational research online, 7(1), 14-48.

Konsortium PISA.ch. (2018). PISA 2015: Schülerinnen und Schüler der Schweiz im internationalen Vergleich. SBFI/EDK und Konsortium PISA.ch. https:/pisa.educa.ch/sites/default/files/uploads/2018/ 11/pisa2015_nationaler-bericht_d.pdf. Zugegriffen: 18. Nov. 2019

Kracke, B., \& Noack, P. (2006). Die Rolle der Eltern für die Berufsorientierung von Jugendlichen. In B. Schuster, H. Kuhn \& H. Uhlendorff (Hrsg.), Entwicklung in sozialen Beziehungen Heranwachsen- 
de in ihrer Auseinandersetzung mit Familie, Freunden und Gesellschaft (S. 169-193). Oldenbourg: Lucius \& Lucius.

Landis, J.R., \& Koch, G.G. (1977). The Measurement of Observer Agreement for Categorical Data. Biometrics, 33(1), 159

Maag Merki, K., Kotthoff, H.-G., \& Emmerich, M. (2009). Projektevaluation „Regionale Bildungslandschaften. Qualitätsentwicklung von Schule und Unterricht in staatlich-kommunalen Verantwortungsgemeinschaften“. http://www.lokale-bildungslandschaften.de/fileadmin/bildungslandschaften/ Fachdiskurs/Forschungsvorhaben/Projektbeschreibung_RBL.pdf. Zugegriffen: 19. Juli 2016

Manitius, V. (2013). Regionalisierung und Gerechtigkeit? Eine Betrachtung auf Chancen und Risiken entlang theoretischer und empirischer Hinweise [Technischen Universität Dortmund. https://eldorado. tu-dortmund.de/bitstream/2003/31154/1/dissertation.pdf. Zugegriffen: 23. Sept. 2019

Manitius, V., Jungermann, A., \& Bos, W. (2015). Regionalisierung als gerechtigkeitsbezogene Unterstützungsstrategie für Schulentwicklung. In T. Coelen, A. J. Heinrich \& A. Million (Hrsg.), Stadtbaustein Bildung (S. 351-361). Wiesbaden: Springer. https://doi.org/10.1007/978-3-658-07314-5_31.

Mayring, P. (2002). Einführung in die qualitative Sozialforschung. Weinheim: Beltz.

Mayring, P. (2008). Qualitative Inhaltsanalyse. Grundlagen und Techniken (10. Aufl.). Weinheim: Beltz.

Mayring, P. (2012). Qualitative Inhaltsanalyse. Ein Beispiel für Mixed Methods. In M. Gläser-Zikuda, T. Seidel, C. Rohlfs, A. Gröschner \& S. Ziegelbauer (Hrsg.), Mixed Methods in der empirischen Bildungsforschung (S. 27-36). Münster: Waxmann.

Rawls, J. (2003). Eine Theorie der Gerechtigkeit (Sonderausg.). Frankfurt am Main: Suhrkamp.

Rolff, H. G. (2014). Auswertung der Evaluationen und Empfehlungen zur Weiterentwicklung der Regionalen Bildungsnetzwerke (RBN)in NRW. https://www.schulministerium.nrw.de/docs/Schulentwicklung/ RegionaleBildNetzwerke/Expertise-II-final.pdf. Zugegriffen: 16. Mai 2019

Schalkhausser, S., \& Franziska, T. (2011). Lokale Bildungslandschaften in Kooperation von Jugendhilfe und Schule [Abschlussbericht]. Deutsches Jugendinstitut e. V. http://www.dji.de/bibs/2011_06_08_ Lokale_Bildungslandschaften_in_Kooperation_von_Jugendhilfe_und_Schule.pdf. Zugegriffen: 23. Mai 2018

Schubert, H., Rädler, M., Schiller, K., \& Schmager, S. (2011). Abschlussbericht der externen Evaluation des Programms „Lebenswelt Schule“ - Evaluationsphase 2010 bis 2011 [Abschlussbericht]. Köln: Fachhochschule.

Sen, A. (2005). Human rights and capabilities. Journal of Human Development, 6(2), 151-166. https://doi. org/10.1080/14649880500120491.

SKBF (2018). Bildungsbericht_Schweiz_2018.pdf. Schweizerische Koordinationsstelle für Bildungsforschung. https://www.skbf-csre.ch/fileadmin/files/pdfs/bildungsberichte/2018/Bildungsbericht_ Schweiz_2018.pdf. Zugegriffen: 14. Okt. 2019

Stolz, H.-J. (2009). Gelingensbedingungen lokaler Bildungslandschaften. Die Perspektive der dezentrierten Ganztagsbildung. In P. Bleckmann \& A. Durdel (Hrsg.), Lokale Bildungslandschaften. Perspektiven für Ganztagsschulen und Kommunen (S. 105-119). Wiesbaden: VS. https://doi.org/10.1007/9783-531-91857-0_8.

Tippelt, R., Hickmann, S., \& Ramczykowski-Elgert, E. (2011). „Neue Brücken bauen...zwischen Generationen, Kulturen und Institutionen “. Ein Programm der Baden-Württemberg Stiftung. Abschließender Evaluationsbericht. München: Institut für Pädagogik Lehrstuhl für Allgemeine Pädagogik und Bildungsforschung Ludwig-Maximilians-Universität.

Wolff, S. (2000). Dokumenten- und Aktenanalyse. In U. Flick, E. v. Kardorff \& I. Steinke (Hrsg.): Qualitative Forschung. Ein Handbuch (S. 502-513). Reinbek: Rowohlt. 